\title{
Li-rich giants in the Galactic Bulge. Is Li linked only to evolutionary status?
}

\author{
Oscar A. Gonzalez ${ }^{1}$ \\ ${ }^{1}$ Southern Observatory, Karl-Schwarzschild-Strasse 2, D-85748 Garching, Germany \\ email: ogonzale@eso.org
}

\begin{abstract}
In our detailed study of chemical abundances in the Galactic bulge (see Zoccali et al. 2008 for a description of the entire project) we have measured Li abundances by fitting synthetic spectra to the ${ }^{7} \mathrm{Li}(6707.18 \AA)$ line for $\sim 400$ giants in Baade's Window and a field at $\mathrm{b}=-6$ (Gonzalez et al. 2009). We have found 13 stars showing strong ${ }^{7} \mathrm{Li}$ lines in complete contrast to the rest of the sample for which only upper limits could be obtained. Our sample is at least 1.2 mag brighter than the expected RGB bump, therefore we interpreted our results as evidence for stars that might have avoided the observed extra-mixing process or undergoing a Li enrichment process not necessarily linked to the RGB bump.
\end{abstract}

Keywords. stars: abundances, late-type - Galaxy: bulge

\section{Introduction}

Even with the increasing number of low mass Li-rich giants found to date, we have not been able to fully understand their origin. The main reason is that is becoming difficult to relate Li-rich stars with a single evolutionary status. Charbonnel \& Balachandran (2000) presented a first evidence for a connection between Li-rich stars and evolutionary status showing that a significant number of Li-rich giants fall close to the RGB bump, where an extra-mixing process is expected to act, affecting abundances of $\mathrm{C},{ }^{12} \mathrm{C} /{ }^{13} \mathrm{C}$ and ${ }^{7} \mathrm{Li}$ (Gratton et al. 2000). In particular, after the RGB bump, ${ }^{7} \mathrm{Li}$ abundances are expected to be close to $\mathrm{A}(\mathrm{Li})=0$ as the material from the convective envelope is brought to higher temperatures in which $\mathrm{Li}$ is expected to be destroyed. However, in a very short stage prior to its destruction, ${ }^{7} \mathrm{Li}$ could be produced by Cameron-Fowler mechanism (Cameron \& Fowler 1971). In complete contrast, the number of Li-rich stars not fulfilling the RGB bump connection has been recently increased (Monaco et al. 2007; Uttenthaler et al. 2007; Gonzalez et al. 2009) requiring stellar models to explain a Li production phase almost at any instance along the giant evolutionary sequence.

\section{Can we really constrain Li-rich giants to a single phenomena?}

While 11 stars in our sample show $\mathrm{A}(\mathrm{Li})$ within 0.8 and 2.3 following a well defined trend with $\mathrm{T}_{\text {eff }}$ (Fig. 1), 2 of them have $\mathrm{A}(\mathrm{Li}) \sim 2.8$ and fall away from this relation. From their location in the CMD (nearly 1 mag. above the red clump), Li-rich stars in our sample are not compatible with a Li production phase in the RGB bump, even considering the Bulge distance spread. However, the Li content in the remaining $\sim 400$ stars is in agreement with an extra-mixing process diluting Li at the RGB bump to a value of $\mathrm{A}(\mathrm{Li}) \sim 0$. Therefore, these Li-rich giants might be stars which have avoided the extramixing at the RGB bump and show the Li abundance expected from standard dilution $(\mathrm{A}(\mathrm{Li}) \sim 1.5)$ which would explain the observed trend between ${ }^{7} \mathrm{Li}$ and $\mathrm{T}_{\text {eff. However, }}$. in this scenario, the problem remains for the two Li-rich stars showing $\mathrm{A}(\mathrm{Li}) \sim 2.8$ for 

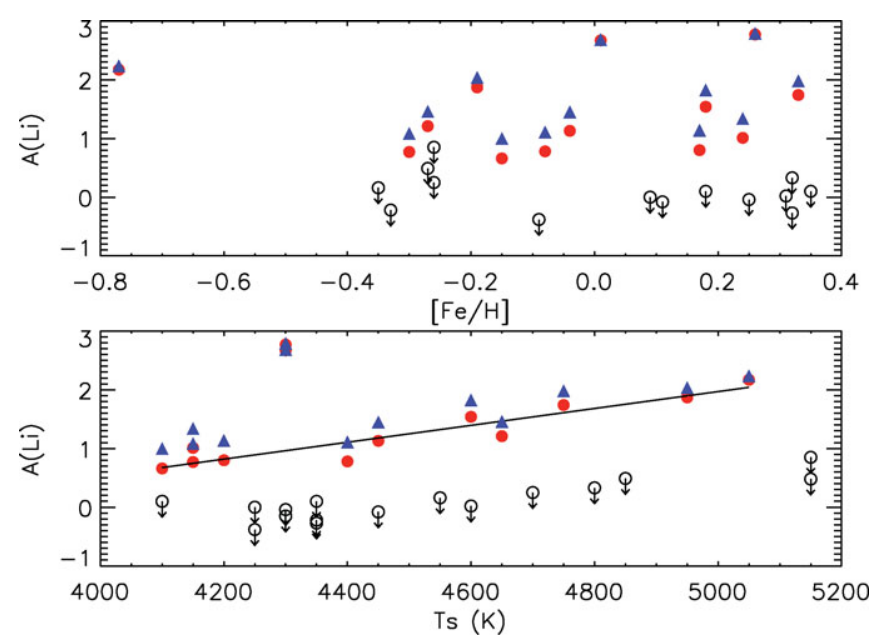

Figure 1. Relation between Li abundances and effective temperature (lower panel). Red filled circles are Li abundances measured under LTE and empty circles are upper limits. Blue triangles are $\mathrm{A}(\mathrm{Li})$ with NLTE corrections. No relation is observed between $\mathrm{A}(\mathrm{Li})$ and metallicity (upper panel).

which the high Li content could only be explained by an undergoing Li production phase. Models have shown that Li evolution in low-mass giants is highly dependent on magnetic field properties (Guandalini et al. 2009). By taking into account these magnetic properties, stars avoiding the extra-mixing process in the RGB bump might be explained (Charbonnel \& Zahn 2007) as well as Li production in other instances of the giant evolution. A different approach must be considered as well, as Li has been probed to be affected by the presence of planets, at least in main sequence stars. In more evolved stars, this might also be the case. The engulfment of a planet would hardly contain enough material to enrich the surface of the star, but according to the models presented by Denissenkov \& Herwig (2004), the addition of angular momentum might be enough to trigger a short $\mathrm{Li}$ production phase which might explain Li enriched giants at any moment during their giant evolution.

In conclusion, as the number of Li-rich stars has increased, it has became clear that their occurrence cannot be confined to a single evolutionary phase. Other parameters such as magnetic field properties or the presence of planets, might also be playing an important role.

\section{References}

Cameron, A. G. W. \& Fowler, W. A. 1971, ApJ, 164, 111

Charbonnel, C. \& Balachandran, S. C. 2000, A\&AS, 359, 563

Charbonnel, C. \& Zahn, J. P. 2007b, A\& A, 476, L29

Denissenkov, P. A. \& Herwig, F. 2004, ApJ, 612, 1081

Gonzalez, O. A., Zoccali, M., Monaco, L., Hill, V. et al. 2009, A\&A, 508, 289

Gratton, R. G., Sneden, C., Carretta, E., \& Bragaglia, A. 2000, A\& $A, 354,169$

Guandalini, R., Palmerini, M., Busso, M., \& Uttenthaler, S. 2009, PASA, 26, 168

Monaco, L., Bellazzini, M., Bonifacio, P., Buzzoni, A., Ferraro, F. R., Marconi, G., Sbordone, L., \& Zaggia, S. 2007, A\&BA, 464, 201

Monaco, L. \& Bonifacio, P. 2008, MemSAI, 79, 1

Uttenthaler, S., Lebzelter, T., Palmerini et al. 2007, A\&SA, 471, L41

Zoccali, M., Lecureur, A., Hill, V. et al. 2008, A\&̈A, 486, 177 